\title{
Evaluation of glycerol encapsulated with alginate and alginate-chitosan polymers in gut environment and its resistance to rumen microbial degradation
}

\author{
Ramadan Gawad $^{1,2}$ and Vivek Fellner ${ }^{1, *}$
}

\author{
* Corresponding Author: Vivek Fellner \\ Tel: +1-919-515-4018, Fax: +1-919-515-4463, \\ E-mail: vfellner@ncsu.edu \\ 'Department of Animal Science and Interdepartmental \\ Nutrition Program, North Carolina State University, \\ Raleigh, NC 27695-7621, USA \\ ${ }^{2}$ Food Technology and Nutrition Division, National \\ Research Center, Dokki, Giza 12622, Egypt \\ ORCID \\ Ramadan Gawad \\ https://orcid.org/0000-0002-7723-568X \\ Vivek Fellner \\ https://orcid.org/0000-0001-6498-7666
}

Submitted Feb 3, 2018; Revised Apr 10, 2018; Accepted Jun 14, 2018
Objective: To determine the effect of gut $\mathrm{pH}$ and rumen microbial fermentation on glycerol encapsulated in alginate and alginate-chitosan polymers.

Methods: Glycerol was encapsulated at $2.5 \%, 5 \%, 7.5 \%$, or $10 \%(\mathrm{w} / \mathrm{w})$ with sodium alginate (A) and alginate-chitosan (AC) polymers. Surface morphology and chemical modifications of the beads were evaluated using scanning electron microscopy and Fourier transform infrared (FTIR) spectra. Encapsulation efficiency was determined at the 5\% glycerol inclusion level in two experiments. In experiment $1,0.5 \mathrm{~g}$ of alginate-glycerol (AG) and alginate-chitosan glycerol (ACG) beads were incubated for $2 \mathrm{~h}$ at $39^{\circ} \mathrm{C}$ in $\mathrm{pH} 2$ buffer followed by $24 \mathrm{~h}$ in $\mathrm{pH}$ 8 buffer to simulate gastric and intestinal conditions, respectively. In experiment 2, $0.5 \mathrm{~g}$ of AG and ACG beads were incubated in $\mathrm{pH} 6$ buffer at $39^{\circ} \mathrm{C}$ for $8 \mathrm{~h}$ to simulate rumen conditions. All incubations were replicated four times. Free glycerol content was determined using a spectrophotometer and used to assess loading capacity and encapsulation efficiency. An in vitro experiment with mixed cultures of rumen microbes was conducted to determine effect of encapsulation on microbial fermentation. Data were analyzed according to a complete block design using the MIXED procedure of SAS (SAS Institute, Cary, NC, USA).

Results: For AG and ACG, loading capacity and efficiency were $64.7 \%, 74.7 \%, 70.3 \%$, and $78.1 \%$, respectively. Based on the FTIR spectra and scanning electron microscopy, ACG treatment demonstrated more intense and stronger ionic bonds. At pH 6, 36.1\% and 29.7\% of glycerol was released from AG and ACG, respectively. At pH 2 minimal glycerol was released but $\mathrm{pH} 8$ resulted in $95.7 \%$ and $93.9 \%$ of glycerol released from AG and ACG, respectively. In vitro microbial data show reduced $(\mathrm{p}<0.05)$ fermentation of encapsulated glycerol after $24 \mathrm{~h}$ of incubation.

Conclusion: The $\mathrm{AC}$ polymer provided greater protection in acidic $\mathrm{pH}$ with a gradual release of intact glycerol when exposed to an alkaline $\mathrm{pH}$.

Keywords: Glycerol; Alginate; Chitosan; Encapsulation; Rumen; pH

\section{INTRODUCTION}

Glycerol is a major byproduct of biodiesel [1]. The increased demand for biodiesel as an alternative energy source has created a large surplus of glycerol [2]. Traditional uses of glycerol are saturated limiting the production of biodiesel as a sustainable source of energy [3]. Glycerol has a high energy content and is recognized as a safe feed ingredient [4]. Low price and surplus quantity makes glycerol economically competitive with other grains as a feed supplement for livestock [5]. However, the amount of glycerol that can be included in the diet is limited because it is rapidly fermented in the rumen which can have a negative effect on microbial fermentation [6,7]. Encapsulation can reduce the amount of glycerol made available in the rumen thereby minimize the negative impact on fermentation and allow greater amounts to 
be included in livestock feeds. Protecting dietary supplements from ruminal environment has been a common approach to allow greater inclusion of supplements and increase nutritional value of the ingredient $[8,9]$. Encapsulating glycerol to bypass rumen fermentation has not previously been investigated.

Our objective was to evaluate alginate and alginate-chitosan as encapsulating polymers for glycerol. Alginate and chitosan are biocompatible, non-toxic and have selective biodegradability [10]. Chitosan is less soluble in alkaline $\mathrm{pH}$ and alginate is insoluble at low $\mathrm{pH}$. We hypothesized that compared to alginate alone the alginate-chitosan mix would result in a stronger matrix and be more effective as an encapsulating polymer.

\section{MATERIALS AND METHODS}

\section{Materials}

Medium viscosity $(\geq 2,000 \mathrm{cp})$ sodium alginate isolated from brown algae, with a molecular weight between 80 and 120 $\mathrm{kDa}$ and a mannuronic to guluronic acid ratio of 1.56 was purchased from Sigma-Aldrich, St. Louis, MO, USA. The polymer chitosan (molecular weight: 190 to $310 \mathrm{kDa}$, deacetylation degree $75 \%$ to $85 \%$, and viscosity of 200 to $800 \mathrm{cp}$ ) was also purchased from Sigma-Aldrich, USA. High purity grade glycerol (99.8\%) and calcium chloride, as a cross linking agent, were purchased from Fisher Scientific, Pittsburgh, PA, USA. All other chemical reagents were of analytical grade.

\section{Preparation of beads}

Glycerol was added at $2.5 \%, 5 \%, 7.5 \%$, or $10 \%$ to each of two bead solutions. One bead solution contained $2.5 \%(\mathrm{w} / \mathrm{v})$ sodium alginate in distilled water. The other bead solution contained a chitosan solution $(1.25 \% \mathrm{w} / \mathrm{v})$ in dilute acetic acid ( $1 \%)$ mixed with sodium alginate $(2.5 \% \mathrm{w} / \mathrm{v})$. The alginateglycerol (AG) and alginate-chitosan glycerol (ACG) beads were prepared by adding the different levels of glycerol into a $500 \mathrm{~mL}$ wide mouth beaker containing the two bead solutions, respectively. The bead solution mixture was allowed to stir at $500 \mathrm{rpm}$ for an hour, then transferred into a separating funnel and dropped through an 18-gauge needle $(38 \mathrm{~mm}$ length, $1.27 \mathrm{~mm}$ diameter) from a $100 \mathrm{~mL}$ glass syringe into a wide mouth beaker containing a $3 \%(\mathrm{w} / \mathrm{v})$ calcium chloride solution. The beads were allowed to mix under gentle stirring (10 rpm) for an hour to harden. After one hour, the beads were filtered through a cheesecloth and rinsed under distilled water. Following adequate rinsing, the beads were air dried at $23^{\circ} \mathrm{C}$ for 12 hours and stored in air tight containers for subsequent experiments.

Effect of glycerol concentration on loading capacity and encapsulation efficiency

Representative samples of AG and ACG beads at each level of glycerol inclusion i.e. $2.5 \%, 5 \%, 7.5 \%$, or $10 \%$ were prepared, in triplicate, to determine free glycerol content according to the method described by Bondioli and Della Bella [11] at 412 nm using a UV spectrophotometer (Shimadzu UV1601, Tokyo, Japan). A calibration curve $\left(\mathrm{R}^{2}=0.996\right)$ was generated from glycerol standard solutions at known concentrations. The loading capacity (LC) and encapsulation efficiency (EE) of the AG and ACG glycerol beads were calculated based on the equations below:

$$
\begin{aligned}
\operatorname{LC}(\%)= & (\text { glycerol content in beads } / \text { weight of beads }) \times 100 \\
\mathrm{EE}(\%)= & (\text { glycerol content in beads } \\
& \quad / \text { initial amount of glycerol added }) \times 100
\end{aligned}
$$

\section{Scanning electron microscopy analysis}

The surface morphology of the beads was observed using a field emission scanning electron microscopy (SEM) (FE-SEM, JEOL, 6400F, Japan). The beads were mounted on an appropriate stub, coated with carbon and gold, and photographs were taken at a magnification of $50 \times, 1,000 \times$, and 10,000 . The working distance was maintained at $20 \mathrm{~mm}$ and the acceleration voltage was $5 \mathrm{kV}$.

\section{Structural analysis by Fourier transform infrared spectroscopy}

Fourier transform infrared (FTIR) spectrum of glycerol, alginate, chitosan, AG, and ACG beads were recorded using a transmittance mode iS50 Thermo Nicolet Nexus 670 FTIR (Thermo Scientific, Waltham, MA, USA) with a built-in diamond crystal. The absorbance pattern of diamond typically seen between 1,900 and 2,400 $\mathrm{cm}^{-1}$ was erased. No bonds were observed in that area. Analysis was performed within the spectral region of 400 to $4,000 \mathrm{~cm}^{-1}$ with 32 scans recorded at $4 \mathrm{~cm}^{-1}$ resolution. FTIR spectra from the different bead preparations were compared to evaluate chemical modifications.

\section{Glycerol release in-vitro}

Glycerol release experiments were performed at $\mathrm{pH}$ 6, 2, and 8 to simulate rumen, gastric, and intestinal conditions, respectively. The gastric buffer ( $\mathrm{pH} 2$ ) was prepared by mixing $50 \mathrm{~mL}$ of a $0.2 \mathrm{M} \mathrm{KCl}$ with $13 \mathrm{~mL}$ of $0.2 \mathrm{M} \mathrm{HCl}$ and brought to a total volume of $200 \mathrm{~mL}$ with distilled water. The intestinal buffer ( $\mathrm{pH} 8$ ) was prepared by mixing $100 \mathrm{~mL}$ of a $0.1 \mathrm{M} \mathrm{KH}_{2} \mathrm{PO}_{4}$ with $93.4 \mathrm{~mL}$ of $0.1 \mathrm{M} \mathrm{NaOH}$ and brought to a total volume of $200 \mathrm{~mL}$ with distilled water and the rumen buffer ( $\mathrm{pH}$ 6) was prepared by mixing $100 \mathrm{~mL}$ of $0.1 \mathrm{M} \mathrm{KH}_{2} \mathrm{PO}_{4}$ with 11.2 $\mathrm{mL}$ of $0.1 \mathrm{M} \mathrm{NaOH}$ and brought to a total volume of $200 \mathrm{~mL}$ with distilled water.

The ability of the encapsulating polymers to protect glycerol from acidic and alkaline hydrolysis was assessed in two separate in vitro experiments. In experiment 1 , acidic and alkaline 
phases were studied sequentially by placing $0.5 \mathrm{~g}$ samples of AG and ACG beads inside conical flasks $(200 \mathrm{~mL})$, containing $100 \mathrm{~mL}$ of $\mathrm{pH} 2$ buffer. Four flasks were prepared per treatment to provide for additional replications. All flasks were incubated at $39^{\circ} \mathrm{C}$ for $2 \mathrm{~h}$ to simulate gastric digestion. After $2 \mathrm{~h}$, a sample was taken and stored for subsequent glycerol analysis. The beads were rinsed with distilled water and transferred to $\mathrm{pH} 8$ buffer at $39^{\circ} \mathrm{C}$ and incubated until $24 \mathrm{~h}$ to simulate intestinal conditions. In experiment $2,0.5 \mathrm{~g}$ samples of AG and ACG beads were incubated in $200 \mathrm{~mL}$ of pH 6 buffer at $39^{\circ} \mathrm{C}$ for $8 \mathrm{~h}$ to simulate rumen conditions. Each treatment was repeated four times to provide for additional replications. Samples were withdrawn at $2 \mathrm{~h}$ intervals and stored for subsequent glycerol analysis. Free glycerol content was determined at $412 \mathrm{~nm}$ using a spectrophotometer [11].

\section{In vitro fermentation using mixed rumen microbes}

In vitro fermentation was conducted using batch cultures of mixed rumen microorganisms. Diets consisted of pelleted alfalfa and corn (60:40). Free (unprotected) glycerol and encapsulated glycerol beads were included to provide glycerol at $25 \%$ of dry matter. Glycerol, AG and ACG were substituted for corn. Treatments included: i) Con (no glycerol), ii) G (free unprotected glycerol), iii) AG (alginate beads), and iv) ACG (alginate-chitosan beads). Donor animal for rumen contents was a non-lactating cannulated Holstein cow fed a predominantly forage diet. Rumen contents were collected $2 \mathrm{~h}$ after the morning feeding and immediately transported to the laboratory in a thermal flask. In the lab, rumen contents were strained through four layers of cheesecloth and mixed with a buffer solution [12] in a 1:2 ratio (vol/vol). The rumen contents and buffer were maintained at $39^{\circ} \mathrm{C}$ and $\mathrm{CO}_{2}$ was purged through the flasks at all times to minimize exposure to $\mathrm{O}_{2}$. Individual treatment substrates were quantitatively weighed into $120 \mathrm{~mL}$ bottles, in triplicate, for each time period and allowed to ferment for 0 and 24 hours. Bottles containing only the rumen inoculum and no substrate served as blanks. Forty $\mathrm{mL}$ of the buffered rumen inoculum were added into each bottle under constant flow of $\mathrm{CO}_{2}$. Bottles were immediately sealed with rubber lined screw caps and placed in a $39^{\circ} \mathrm{C}$ water bath. The $0 \mathrm{~h}$ samples were sealed and immediately placed on ice to stop fermentation. After $24 \mathrm{~h}$, samples were removed from the water bath, placed on ice and culture $\mathrm{pH}$ was recorded prior to storing bottles in a walk in freezer at $-20^{\circ} \mathrm{C}$ for further chemical analysis. Short chain fatty acids (SCFAs) were quantified using a gas chromatograph (Model 3380, Varian, Walnut Creek, CA, USA) using a fused silica capillary column (Nukol; Supelco Inc., Bellefonte, PA, USA). The amount of SCFA in each batch culture after $24 \mathrm{~h}$ of incubation was corrected for the amount of SCFA in the rumen fluid used as inoculum.

\section{Statistical analysis}

Data from the encapsulation experiment were analyzed according to a complete block design using the MIXED procedure of SAS (SAS Institute, Cary, NC, USA). The statistical model included fixed effect of polymers, amount of glycerol and the polymerxglycerol interaction. Replicate was included as the random term. Data from the glycerol release experiments were analyzed according to a complete block design using the MIXED procedure of SAS. The statistical model included the fixed effects of polymer, $\mathrm{pH}$ and incubation time and the respective interactions. Data from the in vitro fermentation with mixed rumen microbes was analyzed by hour ( 0 vs 24 ) as a complete block design. The MIXED procedure of SAS was used to separate means within the hour. The statistical model included the fixed effect of treatment. Replicate was included as random effect. Differences among treatment means in all experiments were determined by the LSmeans/pdiff option. Differences were considered significant at $\mathrm{p}<0.05$ with trends defined as $0.05<\mathrm{p}<0.10$.

\section{RESULTS}

\section{Formation and physical examination of beads}

Images of wet and dry beads are presented in Figure 1. Fresh beads (Figure 1A) were round in shape with a smooth and homogeneous surface. The drying process collapsed the beads and reduced their dimensions (Figure 1B). The loss of water made the beads slightly darker in color and more ellipsoidal with a rougher surface. Both, the AG and ACG beads were similar in color and size. The ionotropic gelation method used in this study to encapsulate glycerol through an 18-gauge needle resulted in fresh bead size of approximately $3 \mathrm{~mm}$ in diameter with a spherical appearance and a smooth outer matrix (Figure 1).

\section{Glycerol loading capacity and encapsulation efficiency}

There was a significant effect of the concentration of glycerol and polymer treatment on glycerol LC (Figure 2). Increasing the concentration of glycerol increased $(p<0.001)$ the amount of glycerol loaded irrespective of the polymer treatment. The greatest increase in LC, irrespective of polymer, occurred when the level of glycerol was raised from $2.5 \%$ to $5 \%$. Further increases in the level of glycerol concentrations ( $7.5 \%$ and $10 \%)$ did not have an appreciable increase in glycerol LC for either AG or ACG treatments. Compared with AG, the ACG treatment significantly increased LC at all glycerol concentrations (Figure 2). The maximum LC was $73.6 \%$ and $66.7 \%$ for ACG and AG beads, respectively, when glycerol was included at $10 \%$.

The EE for the AG and ACG beads is illustrated in Figure 3. The ACG beads resulted in the highest efficiency (78.5\%) compared to the AG beads (72.2\%) at the $2.5 \%$ of glycerol addition. Increasing the glycerol LC resulted in a significant and linear decrease in EE with the ACG treatment; the effect 


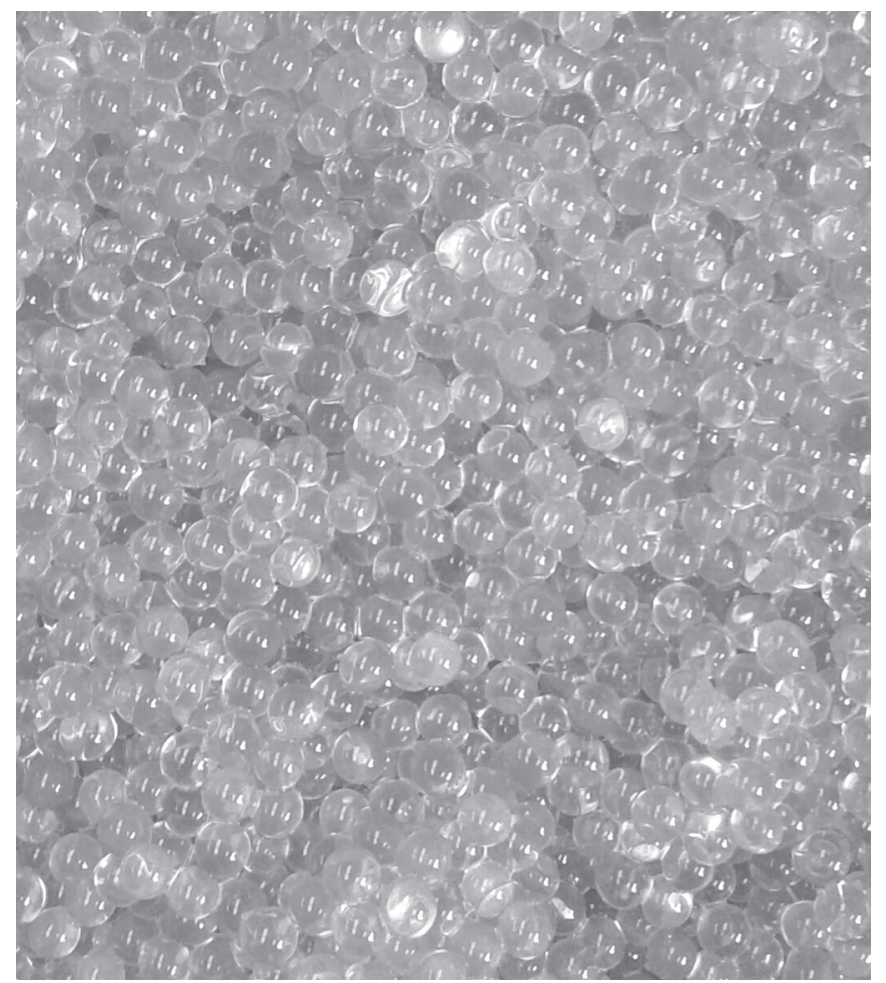

(A)

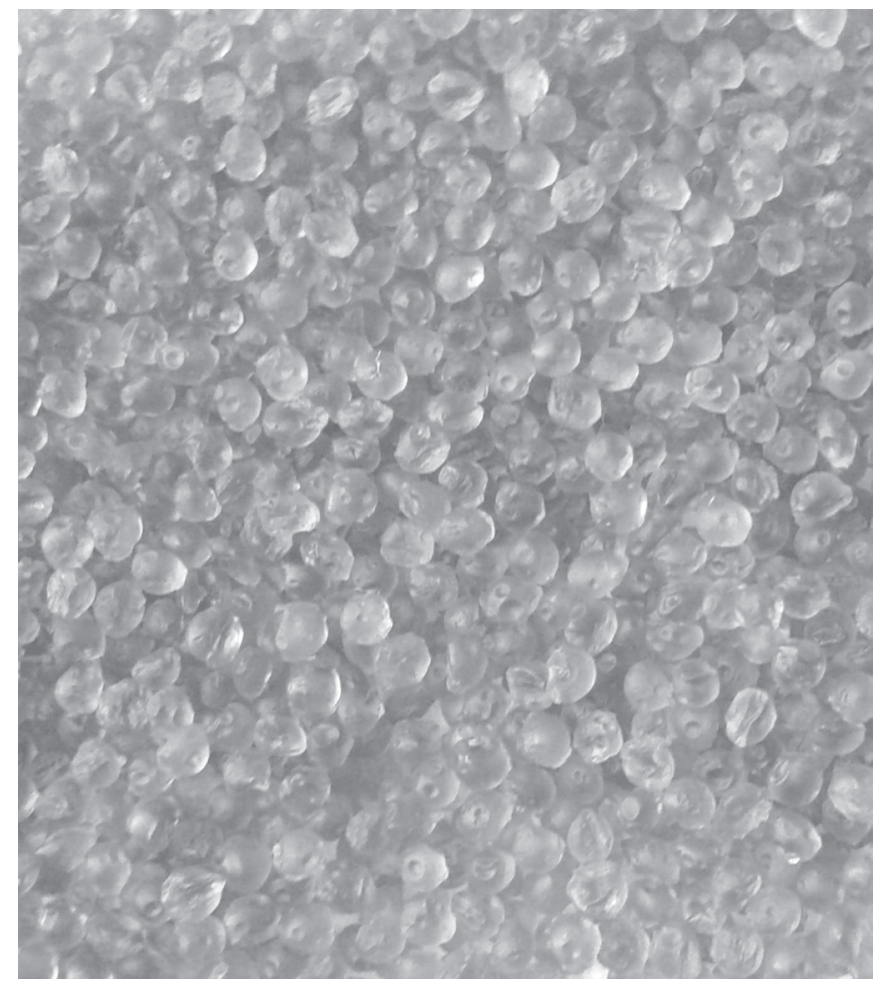

(B)

Figure 1. Glycerol beads ( $\bar{X}$ diameter $=3 \mathrm{~mm}$ ) encapsulated in alginate (AG). Beads were prepared by dripping $5 \%$ (w/v) glycerol and $2.5 \%$ (w/v) sodium alginate into $3 \%(\mathrm{w} / \mathrm{v}) \mathrm{CaCl}_{2}$ solutions. Figure shows photographs of samples taken fresh immediately after filtering through cheesecloth and rinsed in water (A) and samples after drying at $23^{\circ} \mathrm{C}$ for $12 \mathrm{~h}(\mathrm{~B})$.

of AG was variable but significantly lower than the ACG at all glycerol levels.

\section{Scanning electron microscope characterization}

SEM of AG (A, B, C) and ACG (E, F, G) beads are illustrated in Figure 4. To better depict the molecular structure beads

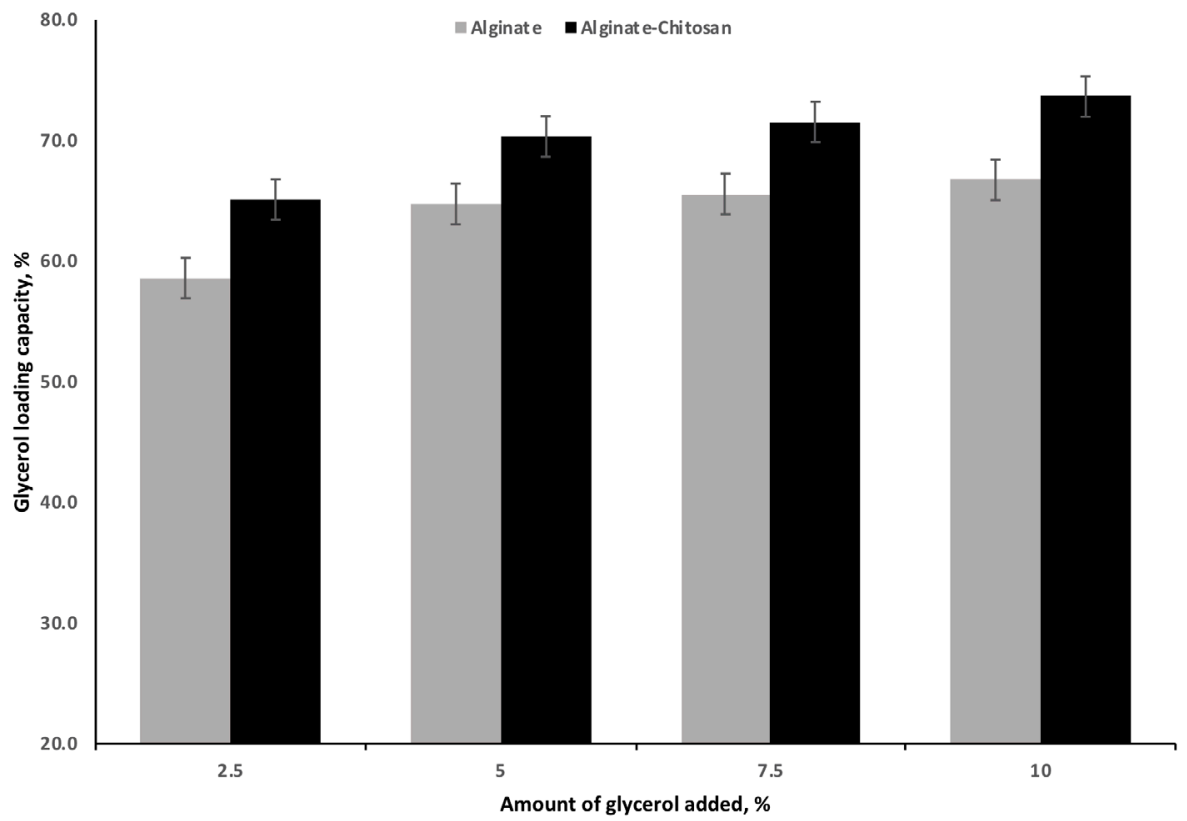

Figure 2. Effect of increasing levels of glycerol on loading capacity of glycerol encapsulated with $2.5 \%(\mathrm{w} / \mathrm{v})$ alginate or $2.5 \%(\mathrm{w} / \mathrm{v})$ alginate and $1.25 \%(\mathrm{w} / \mathrm{v})$ chitosan mixture as encapsulating polymers $(n=3)$. Effect of polymer $(p<0.001)$. Effect of glycerol $(p<0.001)$. 


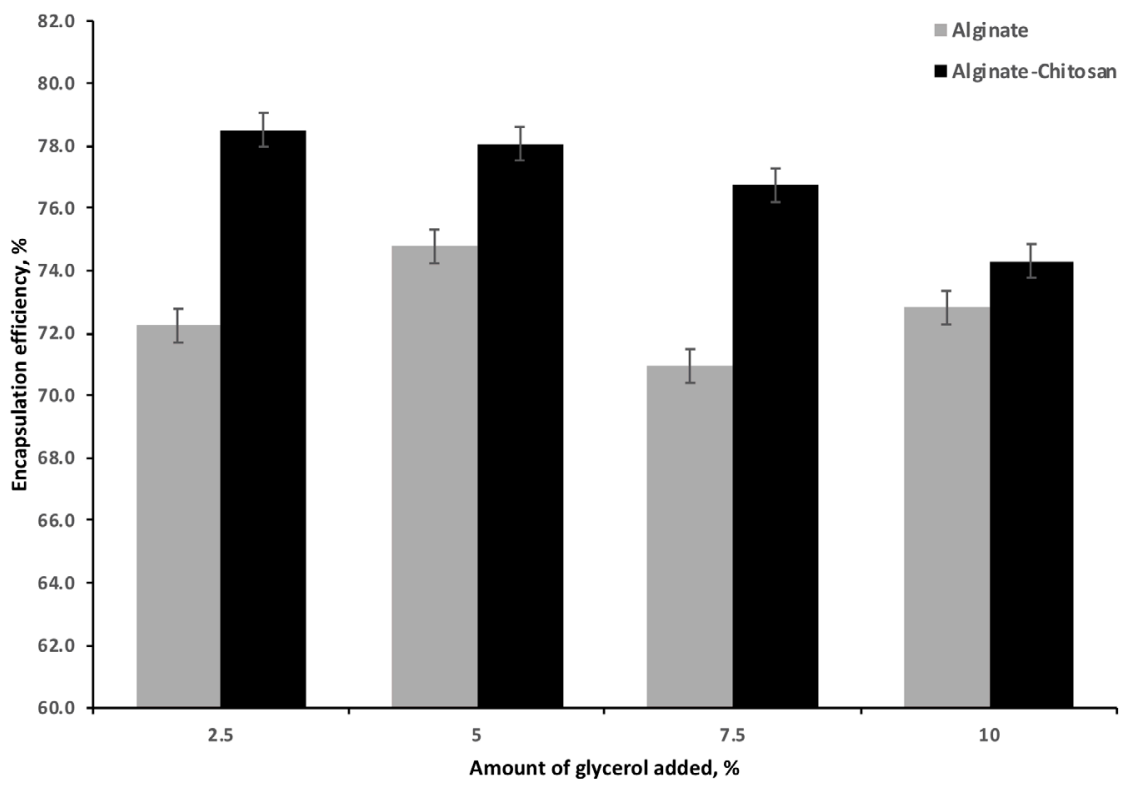

Figure 3. Effect of increasing amounts of glycerol on efficiency of encapsulation by alginate or alginate chitosan polymers $(n=3)$. Effect of polymer $(p<0.001)$. Effect of glycerol $(p<0.001)$. Effect of polymer $\cdot$ glycerol $(p<0.001)$.

were scanned at three intensities $(50 \times, 1,000 \times$, and $10,000 \times)$ as illustrated in A, B, C for the AG beads and E, F, G for the ACG beads. At lower magnification the AG beads (Figure 4A) were smoother and more spherical compared to ACG beads (Figure $4 \mathrm{E}$ ) that had a more rough and undulating surface.
At higher magnification however, we can clearly observe the presence of cracks present in the AG beads (Figure $4 \mathrm{C}$ ) but not in ACG beads (Figure 4G).

\section{Fourier transform infrared analysis}
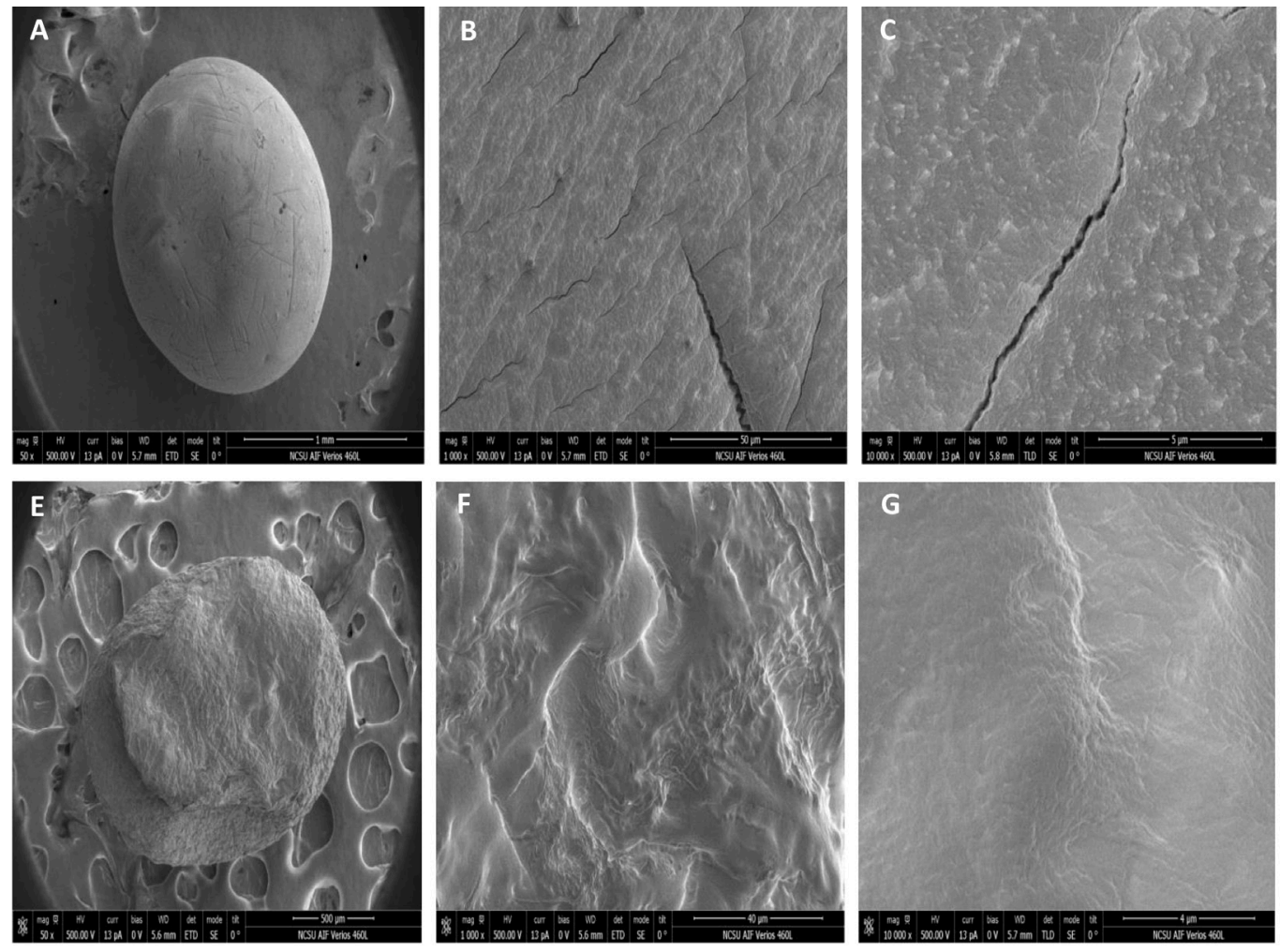

Figure 4. Scanning electron microscopy photographs of glycerol beads encapsulated in alginate (AG) or alginate-chitosan (ACG) polymers. Surface morphology of the bead preparations is depicted at scanning intensity of $50 \times, 1,000 \times$, and $10,000 \times$ for $A G(A, B, C)$ and $A C G(E, F, G)$, respectively. 


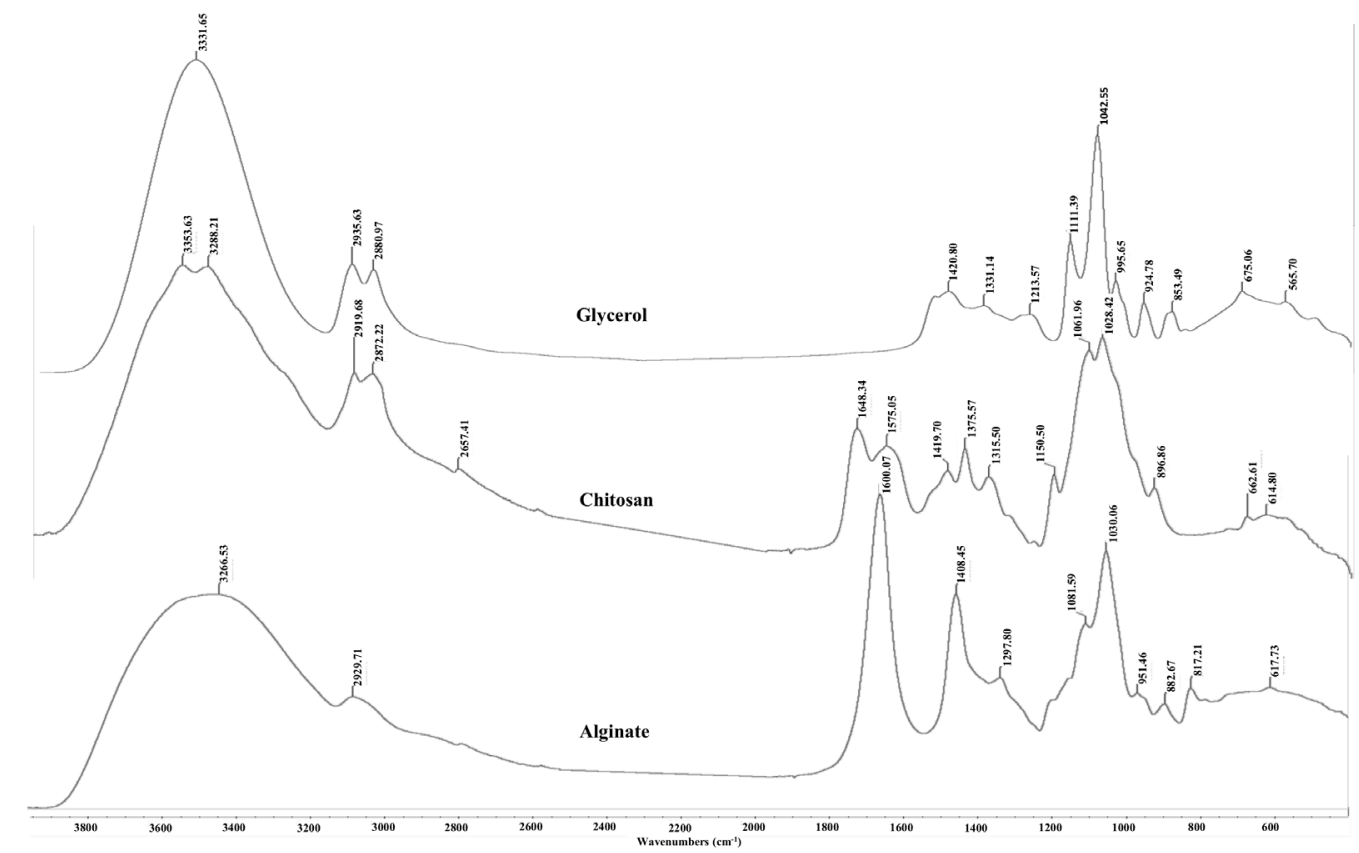

Figure 5. Fourier transform infrared spectra of free glycerol, chitosan and sodium alginate. Peaks characteristic of $\mathrm{OH}$ bonding, $\mathrm{CH}$ and $\mathrm{CO}$ stretching appear at $~ 3,300$

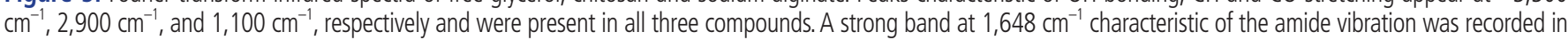
chitosan.

The FTIR spectra of glycerol, alginate, chitosan powder and AG and ACG beads are shown in Figures 5 and 6, respectively. The large peak in glycerol at $3,331.65 \mathrm{~cm}^{-1}$ is due to the characteristic $\mathrm{OH}$ stretching modes. The two peaks at 2,935 and $2,880 \mathrm{~cm}^{-1}$ are reflective of the $\mathrm{CH}$ stretching vibrations of glycerol whereas the peaks between 1,500 and $1,200 \mathrm{~cm}^{-1}$ are due to the $\mathrm{CH}$ bending. The $\mathrm{C}-\mathrm{O}$ stretching is shown in peaks between 1,200 and $900 \mathrm{~cm}^{-1}$. Alginate powder spectrum also shows a broad band at $3,266 \mathrm{~cm}^{-1}$ related to $\mathrm{OH}$ stretching with strong intra and/or inter hydrogen bonding. The peak at $2,929 \mathrm{~cm}^{-1}$ is ascribed to the overlapping symmetrical and asymmetrical C-H stretching vibration of aliphatic chains

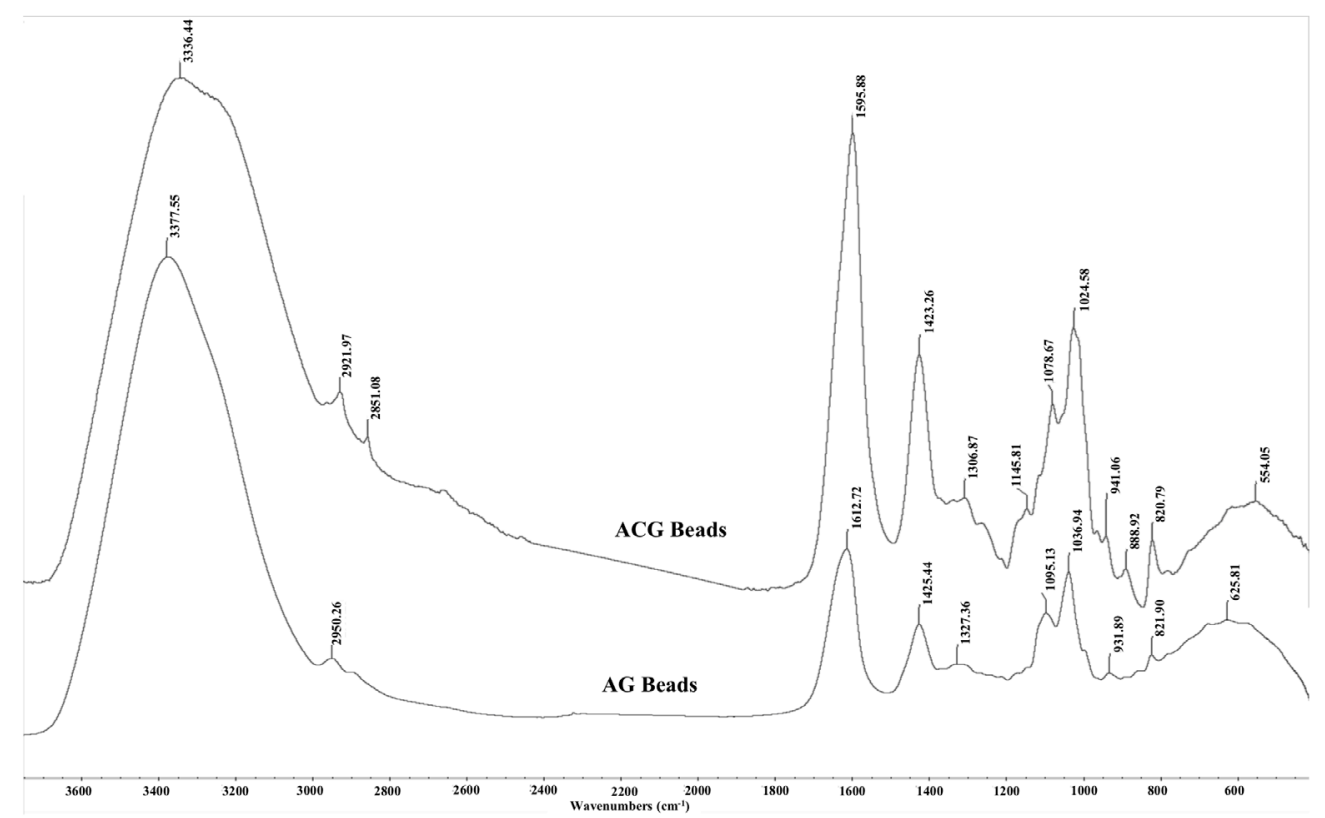

Figure 6. Fourier transform infrared spectra of glycerol encapsulated in alginate (AG) or alginate-chitosan (ACG) polymers. Spectral position of peaks are relatively similar to parent compounds i.e. glycerol, chitosan, and alginate. Greater intensity and narrower peaks in ACG reflect stronger ionic bonds between amine groups in chitosan and carboxylate groups of alginate. 
$\left(-\mathrm{CH}_{2}-,-\mathrm{CH}_{3}\right)$. The asymmetric and symmetric vibrational modes of carboxylate ions $\left(\mathrm{COO}^{-}\right)$were recorded at 1,600 and $1,408 \mathrm{~cm}^{-1}$, respectively. The vibrational mode at $1,081 \mathrm{~cm}^{-1}$ was attributable to the $\mathrm{C}-\mathrm{O}$ stretching vibration of pyranosyl ring. Due to its polysaccharide structure the $(\mathrm{C}-\mathrm{O}-\mathrm{C})$ stretching vibration of sodium alginate was manifested at $1,030 \mathrm{~cm}^{-1}$. The spectrum of chitosan is characterized by broad and intense dimeric bands at 3,353 to $3,288 \mathrm{~cm}^{-1}$ (hydrogen bonded $\mathrm{OH}$ stretching overlapped with $\mathrm{N}-\mathrm{H}$ stretching bands). Chitosan also showed bands at 2,919 and 2,872 $\mathrm{cm}^{-1}$ due to asymmetric stretching of $-\mathrm{CH}_{2}-$ and $-\mathrm{CH}_{3}$ groups. The N-H deformation band of chitosan was found at $1,559 \mathrm{~cm}^{-1}$. The strong bands at 1,648 and $1,575 \mathrm{~cm}^{-1}$ are ascribed to the amide $\mathrm{I}(\mathrm{C}=\mathrm{O}$ stretching) and amide II ( $\mathrm{N}-\mathrm{H}$ bending modes) and $\mathrm{C}-\mathrm{N}$ stretching vibrations of chitosan $[13,14]$. A characteristic peak at $898 \mathrm{~cm}^{-1}$ was observed due to the $\beta-1-4$ glycosidic linkage bond in chitosan.

\section{Release experiments}

Glycerol release from AG and ACG beads when incubated in rumen buffer at $\mathrm{pH} 6$ and $39^{\circ} \mathrm{C}$ for $8 \mathrm{~h}$ is shown in Figure 7. Irrespective of polymer treatment, exposing beads to rumen $\mathrm{pH}$ for $8 \mathrm{~h}$ resulted in a steady release of glycerol. However, the amount of glycerol that escaped from the AG beads was significantly greater when compared to ACG. At end of $8 \mathrm{~h}$, $36.1 \%$ of glycerol was released from the AG beads compared with $29.8 \%$ for the ACG beads.

Glycerol release from AG and ACG beads when incubated at $39^{\circ} \mathrm{C}$ in gastric buffer at $\mathrm{pH} 2$ for $2 \mathrm{~h}$ and then in intestinal buffer at $\mathrm{pH} 8$ until $24 \mathrm{~h}$ is shown in Figure 8 . At the end of $2 \mathrm{~h}$, the amount of glycerol released in gastric buffer tended $(p<0.08)$ to be similar between AG and ACG beads and averaged 15.4\% and $12.6 \%$, respectively. Following gastric conditions, both beads were exposed to intestinal buffer for an additional 22 $\mathrm{h}$. The period from 2 to $6 \mathrm{~h}$ simulated retention time in the small intestine and 12 to $24 \mathrm{~h}$ simulated retention in the large

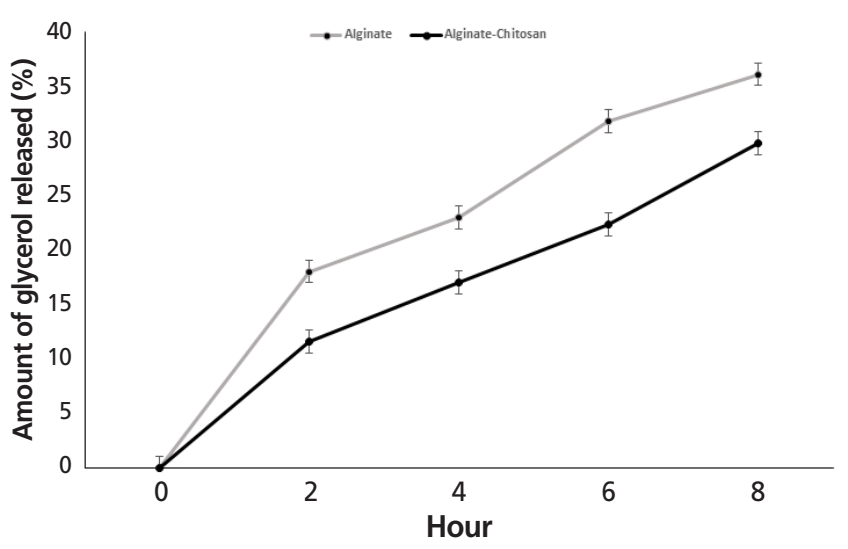

Figure 7. Effect of incubating glycerol encapsulated in alginate (AG) or alginatechitosan (ACG) polymers in a buffer with a pH of 6.0 to simulate glycerol release in the rumen $(n=3)$.

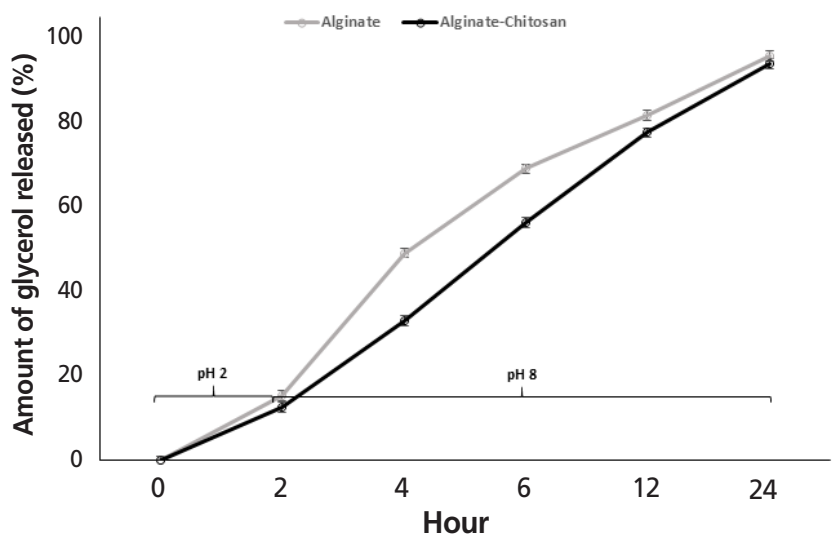

Figure 8. Effect of incubating glycerol encapsulated in alginate (AG) or alginatechitosan (ACG) polymers in a buffer with a pH of 2 and 8 to simulate glycerol release in the gastric and intestine, respectively $(n=3)$.

intestine including colon and cecum of herbivores. During the initial 2 to $6 \mathrm{~h}$ corresponding to the small intestinal retention time, $69 \%$ of the glycerol was released $(\mathrm{p}<0.05)$ from AG compared to $56 \%$ from the ACG beads. However, by $24 \mathrm{~h}$, more than $90 \%$ of the glycerol was released irrespective of the polymer treatment $(\mathrm{p}>0.10)$. At the higher $\mathrm{pH}$ of the intestinal buffer simulating the small and large intestines substantial amounts of glycerol escaped from both the AG and ACG beads and polymer treatment did not have an effect ( $>0.10)$. More than $90 \%$ of the glycerol was released from both AG and ACG beads at the end of $24 \mathrm{~h}$ of incubation in the intestinal buffer. During the initial $6 \mathrm{~h}$ of incubation glycerol release seemed more rapid from the AG beads compared to the ACG beads. This suggests that ACG would result in a gradual supply of glycerol within the small intestine compared to a rapid release rat with the AG beads.

\section{In vitro fermentation with mixed rumen microbes}

There was no difference $(\mathrm{p}>0.10)$ in culture $\mathrm{pH}$ across all treatments at $0 \mathrm{~h}$ (Table 1). As expected, $\mathrm{pH}$ dropped at $24 \mathrm{~h}$ and was similar for all treatments. After $24 \mathrm{~h}$ of incubation, total SCFA increased $(\mathrm{p}<0.05)$ in Con and G. Acetate was significantly reduced in cultures receiving $G$ (free glycerol) when compared with either the Con or AG and ACG treatments. In contrast, molar proportion of propionate were much higher $(p<0.05)$ in cultures receiving $G$ compared to either Con or AG and ACG. The lower acetate and higher propionate resulted in a significantly lower acetate:propionate ratio in cultures receiving free glycerol $(\mathrm{G})$. Visual observations indicated that the beads behaved similar to the particulate fraction and stayed in suspension during the initial stages of fermentation. At the end of $24 \mathrm{~h}$ of incubation some beads remained in suspension while some sank to the bottom with the more dense material. In this study, we did not measure the density of the beads. However, based on the observed behavior of the beads in rumen cultures, we can speculate that the specific gravity of the 
Table 1. Effect of glycerol either unprotected (G) or encapsulated in alginate $(A G)$, and alginate-chitosan (ACG) polymers on in vitro rumen microbial fermentation ( $n=3$ )

\begin{tabular}{|c|c|c|c|c|c|c|}
\hline \multirow{2}{*}{ Items } & \multirow{2}{*}{ Hour } & \multicolumn{4}{|c|}{ Treatment $^{1)}$} & \multirow{2}{*}{ SEM } \\
\hline & & Con & G & AG & ACG & \\
\hline \multirow[t]{2}{*}{ Culture pH } & 0 & 6.24 & 6.22 & 6.19 & 6.14 & 0.037 \\
\hline & 24 & 5.34 & 5.34 & 5.33 & 5.34 & 0.019 \\
\hline \multicolumn{7}{|c|}{ Short chain fatty acids (SCFA) } \\
\hline \multirow[t]{2}{*}{ Total (mM) } & 0 & 47.7 & 47.4 & 49.7 & 49.7 & 2.06 \\
\hline & 24 & $135.5^{\mathrm{a}}$ & $140.0^{\mathrm{a}}$ & $112.7^{b}$ & $116.3^{b}$ & 1.65 \\
\hline \multirow[t]{2}{*}{ Acetate (A, mol \%) } & 0 & 61.3 & 57.5 & 58.2 & 58.1 & 1.64 \\
\hline & 24 & $50.2^{b}$ & $41.9^{c}$ & $51.6^{\mathrm{ab}}$ & $52.6^{\mathrm{a}}$ & 0.68 \\
\hline \multirow[t]{2}{*}{ Propionate (P, mol \%) } & 0 & 22.2 & 22.1 & 21.5 & 21.3 & 1.64 \\
\hline & 24 & $27.4^{c}$ & $32.6^{\mathrm{a}}$ & $28.7^{b}$ & $29.3^{b}$ & 0.47 \\
\hline \multirow[t]{2}{*}{ Butyrate (B, mol \%) } & 0 & 9.0 & 8.9 & 9.4 & 8.5 & 0.61 \\
\hline & 24 & $18.8^{b}$ & $20.4^{a}$ & $15.5^{c}$ & $13.9^{d}$ & 0.25 \\
\hline \multirow[t]{2}{*}{ Valerate (V, mol \%) } & 0 & 3.3 & 4.4 & 4.2 & 4.2 & 0.36 \\
\hline & 24 & $1.9^{b}$ & $3.4^{\mathrm{a}}$ & $2.1^{b}$ & $2.2^{b}$ & 0.14 \\
\hline \multirow[t]{2}{*}{ BCSCFA, mol \% } & 0 & 7.9 & 7.1 & 6.8 & 7.8 & 1.35 \\
\hline & 24 & $1.6^{\mathrm{ab}}$ & $1.5^{b}$ & $1.9^{\mathrm{a}}$ & $1.8^{\mathrm{a}}$ & 0.10 \\
\hline \multirow[t]{2}{*}{$A: P$} & 0 & 2.8 & 2.6 & 2.7 & 2.8 & 0.23 \\
\hline & 24 & $1.8 \mathrm{a}$ & $1.3^{b}$ & $1.8^{\mathrm{a}}$ & $1.8^{\mathrm{a}}$ & 0.05 \\
\hline
\end{tabular}

SEM, standard error of means; BCSCFA, branched-chain SCFA.

${ }^{1)}$ Con, control (no glycerol); $G$, unprotected glycerol; $A G$, glycerol encapsulated in alginate; $A C G$, glycerol encapsulated in alginate-chitosan.

${ }^{a-d}$ Means within a row with different superscripts differ $(p<0.05)$.

beads was greater than 1 and less than 1.5. Beads used in this study were on the average $3 \mathrm{~mm}$ in diameter. Particles $3 \mathrm{~mm}$ in size with specific gravities ranging between 1 and 1.3 are common within the rumen environment [15].

\section{DISCUSSION}

Among several factors, gauge of the needle and concentration of $\mathrm{CaCl}_{2}$, can alter the size, shape and chemical characteristics of beads [16]. Size of beads ultimately is dictated by their intended application. We used an 18-gauge needle to prepare beads with uniformity and that would hold the maximum amount of glycerol yet be reasonably small in size to mix adequately when included in practical livestock diets. The viscosity of glycerol precludes the use of smaller gauge needles without some form of mechanical pressure. The size and appearance of AG and ACG beads were comparable to results reported by other investigators who used similar polymers for encapsulation $[16,17]$. The irregularity and rough surface in alginatechitosan beads is explained by the adhesive properties of chitosan, more rapid ionic interaction and increased polyanionpolycation complex aggregates [18-20].

Factors that affect EE and LC include polymer concentration, crosslinking agent concentration and cross linking time [16]. Studies have reported variable values for LC and EE with alginate alone or in combination with other polymers. Soliman et al [21] used alginate to encapsulate different kinds of essential oils and reported that the alginate microspheres under optimized conditions provided LC of $22 \%$ to $24 \%$ and $\mathrm{EE}$ of $90 \%$ to $94 \%$ for different types of essential oils. Spherical microspheres of theophylline using sodium alginate as the hydrophilic carrier were reported to have entrapment efficiency of $70 \%$ to 93\% [22]. Encapsulation of liquid smoke flavoring with calcium alginate and calcium alginate-chitosan resulted in encapsulation capacities above 96\% [23]. Encapsulation efficiencies for linseed oil and other plant oils with calcium alginate were $88 \%$ and $71 \%$ to $75 \%$, respectively [24]. Based on our results, we were able to optimize conditions to maximize glycerol LC with acceptable EE.

As expected, the AG and ACG beads reflected the FTIR absorbance peaks from their parent molecules i.e. alginate, glycerol and chitosan (Figure 6). The spectral position of peaks identified in the AG and ACG polymer treatments were relatively similar with slight shifts suggesting changes in fundamental structure between the two polymers. The ACG treatment revealed narrower and more intense bands at regions that reflect new hydrogen bonds between chitosan and alginate and stretching of - $\mathrm{COO}$ groups reflecting polyelectrolyte complex formations. The -COO signal is more intense in the ACG beads compared to the AG beads (Figure 6). Greater intensity in the ACG suggest stronger ionic bonds between chitosan amine groups and carboxyl groups of alginate. In the ACG beads, peak bands shifted to a lower energy with an increase in the strength of the ionic interactions. The evidence of the interaction between alginate and chitosan can be seen in the broadening of some bands and increased intensity of others.

Alginate is a common encapsulating polymer used to pro- 
tect bioactive nutrients from gastrointestinal environment and promote delivery to selective locations within the gut $[25,26]$. Alginate has a pKa of 3.5 and is relatively insoluble in acidic $\mathrm{pH}$ making it an attractive biopolymer for minimizing nutrient release in the gastric region of the gut [27]. Furthermore, the calcium-alginate combination has been shown to dominate the bead structure and form "egg-box" junctions acting as cationic bridges that impart stability and prevent penetration of the layer $[18,28]$. However, alginate ionic cross-linkages may have reduced stability as a result of greater solubility in the presence of monovalent cations [29]. The combination of a cationic polymer like chitosan interacts with alginate to modify thermal and functional properties and increase structure stability [18]. The slow release of glycerol in acidic buffer $(\mathrm{pH} 2)$ was as expected since alginate is insoluble at low $\mathrm{pH}$. The greater response with the ACG beads is reflective of the increased stability of the biopolymers and presence of a stronger outer matrix. At higher $\mathrm{pH}$ there was almost complete release of glycerol. The alginate polymer resulted in more rapid release whereas the combination of alginate and chitosan showed a steady rate of glycerol release. These results are consistent with those reported earlier [13,17,23].

Fermentation data with mixed cultures of rumen microbes clearly show that both polymers provided some degree of protection to glycerol from microbial attack. Addition of free glycerol resulted in increased microbial fermentation as evidenced in the increased total SCFA concentration. The decrease in ruminal acetate and increase in propionate is a classic response to the availability of rapidly fermentable energy source. Encapsulating glycerol in alginate or alginate-chitosan resulted in minimal release of glycerol into the rumen culture and maximized glycerol protection from microbial fermentation. Similar protection from rumen microbes was reported for linseed oil encapsulated with alginate and alginate chitosan [30]. Alginate and chitosan biopolymers have been extensively used to protect delivery of pharmaceutical drugs in the gastrointestinal tract but limited use is reported in the ruminal environment.

Our data show that encapsulation of glycerol with alginate or alginate-chitosan can reduce the amount of free glycerol released in a pH 6 buffer and increase amount of glycerol delivered to the lower digestive tract.

Compared with alginate, the alginate-chitosan mix was more effective in increasing stability of the molecule in all intestinal environments. The LC and EE of glycerol was greater with the alginate-chitosan mix than alginate alone. In vitro fermentation with mixed cultures of rumen microbes further demonstrate the viability of the alginate-chitosan mix to provide protection for glycerol from microbial attack. In vivo experiments will need to be conducted to determine if indeed the combination of alginate-chitosan polymer can result in greater and steady amounts of glycerol delivery to the lower gut.

\section{CONFLICT OF INTEREST}

We certify that there is no conflict of interest with any financial organization regarding the material discussed in the manuscript.

\section{ACKNOWLEDGMENTS}

The authors acknowledge Sarah Jo McLeod for her technical assistance and the support provided by the North Carolina Agricultural Experiment Station. This work was performed in part at the Analytical Instrumentation Facility (AIF) at North Carolina State University, which is supported by the State of North Carolina and the National Science Foundation (award number ECCS-1542015). The AIF is a member of the North Carolina Research Triangle Nanotechnology Network (RTNN), a site in the National Nanotechnology Coordinated Infrastructure (NNCI).

Financial support was provided by the North Carolina Agricultural Experiment Station and the Egyptian Cultural Affairs and Mission Sector, Ministry of Higher Education and Scientific Research, Egypt.

\section{REFERENCES}

1. Thompson JC, He B. Characterization of crude glycerol from biodiesel production from multiple feedstocks. Appl Eng Agric 2006;22:261-5.

2. Crandall L. Glycerol abundance cause for concern. Inform 2004;15:146-7.

3. Gholami Z, Abdullah AZ, Lee K-T. Dealing with the surplus of glycerol production from biodiesel industry through catalytic upgrading to polyglycerols and other value-added products. Renew Sustain Energy Rev 2014;39:327-41.

4. Food and Drug Administration, Code of Federal Regulations, 2006; 21CFR582.1320, Title 21;6. Silver Spring, MD, USA: U.S. Food and Drug Administration; 2006.

5. Donkin SS, Koser SL, White HM, Doane PH, Cecava MJ. Feeding value of glycerol as a replacement for corn grain in rations fed to lactating dairy cows. J Dairy Sci 2009;92:5111-9.

6. Roger V, Fonty G, Andre C, Gouet P. Effects of glycerol on the growth, adhesion, and cellulolytic activity of rumen cellulolytic bacteria and anaerobic fungi. Curr Microbiol 1992;25:197-201.

7. Shin JH, Wang D, Kim SC, Adesogan AT, Staples CR. Effects of feeding crude glycerin on performance and ruminal kinetics of lactating Holstein cows fed corn silage- or cottonseed hullbased, low-fiber diets. J Dairy Sci 2012;95:4006-16.

8. Perfield JW, Lock AL, Pfeiffer AM, Bauman DE. Effects of amide-protected and lipid-encapsulated conjugated linoleic acid (CLA) supplements on milk fat synthesis. J Dairy Sci 2004; 
87:3010-6.

9. Santschi DE, Berthiaume R, Matte JJ, Mustafa AF, Girard CL. Fate of supplementary B-vitamins in the gastrointestinal tract of dairy cows. J Dairy Sci 2005;88:2043-54.

10. van Cleef EHC, Almeida MTC, Perez HL, et al. Crude glycerin changes ruminal parameters, in vitro greenhouse gas profile, and bacterial fractions of beef cattle. Livest Sci 2015;178:15864.

11. Bondioli P, Della Bella L. An alternative spectrophotometric method for the determination of free glycerol in biodiesel. Eur J Lipid Sci Technol 2005;107:153-7.

12. Goering HK, van Soest PJ. Forage fiber analyses (apparatus, reagents, procedures, and some applications). Washington, USA: U.S. Agricultural Research Service; 1970. USDA Agriculture Handbook No. 379.

13. Han J, Guenier A-S, Salmieri S, Lacroix M. Alginate and chitosan functionalization for micronutrient encapsulation. J Agric Food Chem 2008;56:2528-35.

14. Sarmento B, Ferreira D, Veiga F, Ribeiro A. Characterization of insulin-loaded alginate nanoparticles produced by ionotropic pre-gelation through DSC and FTIR studies. Carbohydr Polym 2006;66:1-7.

15. Siciliano-Jones J, Murphy M. Passage of inert particles varying in length and specific gravity through the postruminal digestive tract of steers. J Dairy Sci 1986;69:2304-11.

16. Mandal S, Kumar SS, Krishnamoorthy B, Basu SK. Development and evaluation of calcium alginate beads prepared by sequential and simultaneous methods. Braz J Pharm Sci 2010; 46:784-93.

17. Takka S, Gurel A. Evaluation of chitosan/alginate beads using experimental design:formulation and in vitro characterization. AAPS PharmSci Tech 2010;11:460-6.

18. Segale L, Giovannelli L, Mannina P, Pattarino F. Calcium alginate and calcium alginate-chitosan beads containing celecoxib solubilized in a self-emulsifying phase. Scientifica 2016;Article ID 5062706.

19. Cekić ND, Milić JR, Savić SD, et al. Influence of the preparation procedure and chitosan type on physicochemical properties and release behavior of alginate-chitosan microparticles. Drug
Dev Ind Pharm 2009;35:1092-102.

20. Kulig D, Zimoch-Korzycka A, Jarmoluk A, Marycz, K. Study on alginate-chitosan complex formed with different polymers ratio. Polymers 2016;8:167.

21. Soliman EA, El-Moghazy AY, El-Din MSM, Massoud MA. Microencapsulation of essential oils within alginate: Formulation and evaluation of antifungal activity. J Encapsulation Adsorp Sci 2013;3:48-55.

22. Soni ML, Kumar M, Namdeo KP. Sodium alginate microspheres for extending drug release: formulation and in vitro evaluation. Int J Drug Deliv 2010;2:64-8.

23. Petzold G, Gianelli MP, Bugueno G, et al. Encapsulation of liquid smoke flavoring in ca-alginate and ca-alginate-chitosan beads. J Food Sci Technol 2014;51:183-90.

24. Lopez M, Maudhuit A, Pascual-Villalobos MJ, Poncelet D. Development of formulations to improve the controlled-release of linalool to be applied as an insecticide. J Agric Food Chem 2012;60:1187-92.

25. Lucinda-Silva RM, Salgado HRN, Evangelista RC. Alginatechitosan systems: in vitro controlled release of triamcinolone and in vivo gastrointestinal transit. Carbohydr Polym 2010;81: 260-8.

26. Pongjanyakul T, Rongthong T. Enhanced entrapment efficiency and modulated drug release of alginate beads loaded with drug-clay intercalated complexes as microreservoirs. Carbohydr Polym 2010;81:409-19.

27. Chan AW, Neufeld RJ. Modeling the controllable pH-responsive swelling and pore size of networked alginate based biomaterials. Biomaterials 2009;30:6119-29.

28. George M, Abraham TE. Polyionic hydrocolloids for the intestinal delivery of protein drugs: alginate and chitosan - a review. J Control Release 2006;114:1-14.

29. Lee KY, Mooney DJ. Alginate: properties and biomedical applications. Prog Polym Sci 2012;37:106-26.

30. Gawad RMA, Strable M, Abo El-Nor SA, et al. Encapsulation method to protect unsaturated fatty acids from rumen biohydrogenation in vitro. J Innov Pharmaceuticals Biol Sci 2015; 2:240-51. 